\title{
Impact of Fluidic Chevrons on Jet Noise
}

\author{
Brenda S. Henderson ${ }^{*}$ and Kevin W. Kinzie ${ }^{\dagger}$ \\ NASA Langley Research Center, Hampton, VA 23606 \\ and \\ Julia Whitmire and Amal Abeysinghe ${ }^{\ddagger}$ \\ Goodrich Aerostructures, Chula Vista, CA 91910
}

\begin{abstract}
The impact of alternating fluidic core chevrons on the production of jet noise is investigated. Core nozzles for a representative $1 / 9^{\text {th }}$ scale, bypass ratio 5 model system were manufactured with slots cut near the trailing edges to allow for air injection into the core and fan streams. The injectors followed an alternating pattern around the nozzle perimeter so that the injection alternated between injection into the core stream and injection into the fan stream. For the takeoff condition and a forward flight Mach number of 0.10, the overall sound pressure levels at the peak jet noise angle decrease with increasing injection pressure. Sound pressure levels increase for observation angles less than $110^{\circ}$ at higher injection pressures due to increases in high frequency noise. Greater increases in high frequency noise are observed when the number of injectors increases from 8 to 12. When the forward flight Mach number is increased to 0.28 , jet noise reduction (relative to the baseline) is observed at aft angles for increasing injection pressure while significant increases in jet noise are observed at forward observation angles due to substantial acoustic radiation at high frequencies. A comparison between inflow and alternating injectors shows that, for equal mass injection rates, the inflow nozzle produces greater low frequency noise reduction (relative to the baseline) than the alternating injectors at $90^{\circ}$ and aft observation angles and a forward flight Mach number of 0.28 . Preliminary computational fluid dynamic simulations indicate that the spatial decay rate of the hot potential core flow is less for the inflow nozzle than for the alternating nozzles which indicates that gentle mixing may be preferred over sever mixing when fluidic chevrons are used for jet noise reduction.
\end{abstract}

\section{Introduction}

$\mathrm{E}$ FFECTIVE jet noise reduction methods are needed for commercial turbo-fan engines. Many passive devices, such as chevrons or tabs, reduce noise through enhanced mixing of the jet plume. Passive devices cannot adapt to changes in the flow environment so adjustments cannot be made to compensate for changing flight operations or installation effects. Active mixing devices are particularly attractive as they have the potential to optimize jet noise reduction throughout flight operations and can be deployed only when jet noise reduction is necessary and accompanying performance penalties can be tolerated. The fluidic chevron, which uses air injected near the trailing edge of the nozzle to simulate the mixing characteristics of mechanical chevrons, has the potential for active control. This study investigates the impact of alternating fluidic chevrons on the production of jet noise. Alternating fluidic chevrons are produced by injecting air into the core and fan streams near the trailing edge of the core nozzle. Comparisons are made between the acoustic characteristics of alternating fluidic chevrons and fluidic chevrons produced by injecting air only into the core stream flow.

Mechanical chevrons and tabs are created by cutting serrations in the trailing edge of a nozzle and deflecting the serrations into the flow. These devices mix the streams and result in a reduced volume of high-speed flow. When properly designed, tabs and chevrons reduce low frequency noise and do not significantly increase high frequency noise ${ }^{1,2,3}$. The number of chevrons or tabs, the serration geometry, and the penetration depth of the mixers as well as

\footnotetext{
* Researcher, Aeroacoustics Branch, MS 166, Hampton, VA 23606, AIAA Member.

${ }^{\dagger}$ Researcher, Aeroacoustics Branch, MS 166, Hampton, VA 23606, Senior AIAA Member.

${ }^{\ddagger}$ Insert Job Title, Department Name, Address/Mail Stop, and AIAA Member Grade for third author.
} 
many other factors affect the acoustic radiation resulting from the chevron or tabbed nozzle. Computational fluid dynamic simulations of the flow fields associated with chevron and tab nozzles ${ }^{4}$ show that significant off axis mixing occurs for both types of mixers. Comparisons between numerical results and acoustic measurements indicate that some of the most aggressive mixers produce unacceptable levels of high frequency noise.

Another type of device that has been successful in reducing jet noise in subsonic and supersonic flows is microjet fluidic injection ${ }^{5,6}$. Microjet injection is achieved through the injection of high-pressure fluid at, or slightly downstream of, the core nozzle trailing edge through small injection nozzles. Nitrogen, water, and water saturated with a long-chain polymer have been used for the injection fluid. A shortcoming of this approach is that large mass flows, on the order of $20 \%$ to $50 \%$ of the core mass flow, may be needed to achieve 2 to $3 \mathrm{~dB}$ reduction in overall sound pressure levels at the peak jet noise angle.

A different jet noise reduction concept from that of microjet fluidic injection is the fluidic chevron ${ }^{7}$. Fluidic chevrons are achieved by injecting air through slots cut in the core nozzle near the nozzle trailing edge. The air is injected at a much lower pressure than that used by microjet injection and much lower injection mass flow rates are used to achieve noise reduction. Core fluidic chevrons can be configured so that the injected air is directed only into the core stream (inflow injectors) or alternates in the between flow injected in the core stream and flow injected in the fan stream as the slots are located around the core nozzle perimeter. Preliminary studies with inflow fluidic chevrons indicate that these types of mixers reduce overall sound pressure levels over that of a round nozzle as a result of reductions in low frequency noise. However, increases in high frequency noise are also produced by these types of chevrons.

The study reported here investigates the impact of alternating core fluidic chevrons on the production of subsonic jet noise in a representative bypass ratio 5 laboratory model system. The study investigates the effects of injector number and injection pressure on the radiated noise using computational fluid dynamic simulations and experiments. The results from the alternating fluidic chevrons are compared to those of the inflow fluidic chevrons reported by Kinzie, Henderson, Whitmire, and Abeysinghe ${ }^{7}$.

\section{Experimental Approach}

The experiments were conducted in the Low Speed Aeroacoustics Wind Tunnel (LSAWT) at NASA Langley Research Center. A schematic of the facility is shown in Fig. 1. The LSAWT is a continuous flow, in-draft wind tunnel that provides a free jet for forward flight effects that surrounds the Jet Engine Simulator (JES). The free jet exhausts through a $1.43 \mathrm{~m}$ square nozzle into a $10.36 \mathrm{~m}$ long test cell with a $5.18 \mathrm{~m}$ x $5.18 \mathrm{~m}$ cross section. The floor, ceiling, and walls of the test cell are treated with fiberglass wedges. All test section dimensions are measured from wedge tip to wedge tip. A downstream, acoustically treated flow collector is used to reduce flow recirculation in the test cell.

The JES consists of two independently controlled coannular air streams used to accurately simulate engine nozzles systems. Each stream is equipped with a propane-fired, sudden-expansion burner and an electric pre-heater. A maximum mass flow rate of $7.7 \mathrm{~kg} / \mathrm{sec}$ can be achieved in both streams. Temperature and pressure rakes positioned just upstream of the nozzle contraction are used to measure nozzle operating conditions.

A representative $1 / 9^{\text {th }}$ scale, bypass ratio (BPR) 5 model system was used in the experiments. The model consists of an externally plugged, $12.8 \mathrm{~cm}$ diameter core nozzle with an exit area of $69.4 \mathrm{~cm}^{2}$ and a $23.9 \mathrm{~cm}$ diameter fan nozzle with an exit area of $173.4 \mathrm{~cm}^{2}$ shown schematically in Fig. 2. For the fluidic chevron study, the model system includes a pylon clocked at an angle of $135^{\circ}$ to the microphone array axis.

Acoustic measurements are made with a 28-element sideline microphone array (see Fig.1) located at $3.52 \mathrm{~m}$ from the centerline axis of the JES. The $6.35 \mathrm{~mm}$ diameter condenser microphones (Bruel Kjaer type 4939) are used with the grid caps removed and calibrated with a piston phone and electrostatic calibrator. One-third octave and narrowband data include corrections for microphone calibration, wind tunnel background noise, and atmospheric absorption (corrected to the same reference day using the Shields and Bass ${ }^{8}$ method). For the one-third octave data, the Amiet ${ }^{9}$ point source correction is used to account for acoustic propagation through the free jet shear layer and the data is scaled to full scale and reported at a sideline distance of $543 \mathrm{~m}$. A Doppler shift is used for spectral data.

The fluidic chevron nozzles were used on the core nozzle only. Small slots cut near the trailing edges of the nozzle connected to a common plenum within the nozzle through small internal passages in the nozzle. Air was delivered to the common plenum through an air delivery tube embedded in the pylon (see Fig. 3). In the majority of the work reported here, the fluidic chevrons were alternating so that the air injection alternated around the nozzle perimeter between slots directing the flow into the fan stream and slots directing the flow into the core stream. The 
injection total pressure was measured throughout the experiments and controlled through an upstream regulator. The temperature of the air within the nozzle plenum was recorded. The trailing edges of the core nozzles were extended axially with a 0.005 " thick shim 0.4 " long to prevent the production of discrete tones associated with the thick nozzle trailing edge (see Henderson, Kinzie and Haskin ${ }^{10}$ and Kinzie, Henderson, Whitmire and Abeysinghe ${ }^{7}$ ). Two alternating nozzles were tested, one with 12 twelve injection ports (designated 12A) and one with 8 injection ports (designated 8A).

Data were acquired at representative takeoff and cutback conditions although only data for takeoff conditions with forward flight Mach numbers equal to 0.1 and 0.28 are presented here. In the experiments, the core nozzle pressure ratio (NPR), the ratio of the stagnation pressure to the ambient static pressure, was equal to 1.56 and the total temperature was $828{ }^{\circ} \mathrm{K}$. The fan NPR was equal to 1.75 and the total temperature was $359^{\circ} \mathrm{K}$.

\section{Results}

\section{A. Numerical Results}

Computational fluid dynamic (CFD) simulations were performed on the fluidic chevron nozzles before beginning the fabrication process. The purpose of the simulations was to determine the effect of injection parameters on the mixing characteristics of the nozzles and to compare the general flow characteristics of the fluidic chevrons with those of the mechanical chevrons. While it is not possible to determine the acoustic radiation from the flow field solutions, the numerical results were used to design nozzles that would result in a range of mixing characteristics to determine the impact of the fluidic chevron parameters on the production of jet noise.

The computations were performed with the multi-block, parallel, structured code PAB3D ${ }^{11}$. The flow through the nozzle and plume were simulated by solving the asymptotically steady, compressible, Reynolds-averaged, Navier-Stokes equations using an implicit, upwind, flux-difference splitting finite volume scheme. A standard twoequation k-epsilon model with linear stress representation was used for the turbulence model. The computational domain extended over 30 core nozzle diameters, Dc, downstream of the fan nozzle exit in the streamwise direction and 6 core diameters radially from the centerline. For each configuration, only a sector of the flow field was modeled with symmetry boundary conditions used in the azimuthal direction. Although a pylon was used with all configurations in the experiments, the simulations were performed without a pylon.

The internal injection flow through the core nozzle was not modeled in the simulations. The effect of the injection air flow was modeled as boundary conditions on the portions of the inner core nozzle surface where the injection exhausted from the core nozzle. While the injection port model simplified the computations, the injection mass flow was overestimated due to the neglect of flow passage effects.

The contour plots in Fig. 4 show the total temperature in a plane through the centerline of the jet for a normalized injection pressure ratio, IPR*, of 0.36 . For proprietary reasons, the normalized injection ratio is given by the ratio of the injection stagnation pressure to the pressure at the nozzle lip and normalized by the maximum pressure ratio used in the experiments. The maximum pressure ratio was the same for all experiments. The streamwise distance downstream of the fan nozzle exit,x, has been normalized by the core nozzle diameter, Dc, in the figure. The baseline core nozzle is a round nozzle with no fluidic chevrons. The alternating fluidic chevrons are compared to the inflow fluidic chevron nozzle (6I nozzle) that resulted in the largest jet noise reductions (see Kinzie, Henderson, Whitmire, and Abeysinghe ${ }^{7}$ ). A comparison between the baseline nozzle in Fig. 4 a) and the fluidic chevron nozzles in Figs. 4 a) through d) shows the streamwise extent of the hotter potential core flow exhausting from the core nozzle. The enhanced mixing associated with the fluidic chevrons is shown as a reduction in the streamwise length of the hot core flow from that of the baseline nozzle. Both alternating configurations resulted in a streamwise reduction of the hot potential core flow over that of the baseline nozzle and the inflow fluidic chevron nozzle.

A plot of the centerline temperature as a function of normalized streamwise distance downstream of the fan nozzle exit, $\mathrm{x} / \mathrm{Dc}$, is shown in Fig. 5. The potential core of the hot core flow is shown to extend to approximately $\mathrm{x} / \mathrm{Dc}=13$ for the baseline nozzle, $\mathrm{x} / \mathrm{Dc}=6$ for the $6 \mathrm{I}$ nozzle, $\mathrm{x} / \mathrm{Dc}=5$ for the $12 \mathrm{~A}$ nozzle, and $\mathrm{x} / \mathrm{Dc}=4$ for the $8 \mathrm{~A}$ nozzle. Note that while the $6 \mathrm{I}$ nozzle provides the slowest spatial centerline temperature decay rates compared to those of the alternating configurations, it will be shown later that the 6I nozzle produces the best acoustic characteristics of all of the fluidic chevrons tested.

\section{B. Acoustic}

The narrowband acoustic spectra associated with the injector flow without core and fan streams operating are shown in Fig. 6 for the 12A, 8A, and 6I nozzles at an IPR* $=0.36$. All observation angles are measured from the upstream jet axis. As shown in the figure, the noise produced by the injectors alone (self noise) is relatively 
insensitive to injector configuration for the two alternating nozzles tested. The injector noise of the 6I nozzle is significantly lower than that of the $12 \mathrm{~A}$ and $8 \mathrm{~A}$ nozzles for all frequencies at an observation angle of $90^{\circ}$. The injector noise of the $6 \mathrm{I}$ nozzle is significantly lower than that of the $12 \mathrm{~A}$ and $8 \mathrm{~A}$ nozzles at frequencies except those in the range of $2000 \mathrm{~Hz}$ to $8000 \mathrm{~Hz}$ for an observation angle of $140^{\circ}$. However, the injection mass flow of the 6I nozzle was roughly $50 \%$ of that of the $12 \mathrm{~A}$ nozzle. When compared on an equal injection mass flow basis instead of equal injection pressure ratio, the sound pressure levels produced by the $6 \mathrm{I}$ nozzle at observation angles of $90^{\circ}$ and $140^{\circ}$ were above those of the $12 \mathrm{~A}$ and $8 \mathrm{~A}$ nozzles at all measured frequencies. In a real engine application, it is important to obtain the largest reductions in jet noise with the least injection mass flow rates.

The effect of injection pressure ratio on the sideline directivity at a forward flight Mach number of 0.10 is shown in Figs. 7 and 8 for the 12A nozzle. As the injection pressure ratio is increased from no injection flow (IPR* $=0)$ to an injection pressure ratio of 0.82 , decreases in overall sound pressure levels are observed at aft angles. Increases in overall sound pressure levels over that of the no injection case are observed for observation angle less than $110^{\circ}$ and for normalized injection pressure ratios greater than, or equal to, 0.82 . The acoustic spectra in Fig. $8 \mathrm{~b}$ ) show that reductions in overall sound pressure levels with increasing IPR* at aft observation angles are the result of reduced low frequency noise. The increase in acoustic radiation for the highest injection pressure ratio $\left(\mathrm{IPR}^{*}=0.82\right)$ at angles less than $110^{\circ}$ is the result of significant increases in high frequency noise over that produced by the no injection case [see Fig. 8 a)].

The effect of increasing the forward flight Mach number to 0.28 on the sideline directivity produced by the 12A nozzle is shown in Figs. 9 and 10. A reduction in overall sound pressure levels from that associated with the no injection case is observed for angles greater than $110^{\circ}$ and for an injection pressure ratio of 0.82 . Significant increases in acoustic radiation over that of the no injection case are observed for IPR $*=0.82$ and observation angles less than $110^{\circ}$. Unlike the data for the forward flight Mach number of 0.10 , the sideline directivity for IPR* $=0$ and $\mathrm{IPR}^{*}=0.27$ are quite similar. The acoustic spectra in Figs. $10 \mathrm{a}$ ) and b) show that increasing the injection pressure ratio to 0.82 significantly impacts the low frequency noise at aft observation angles and the high frequency noise at $90^{\circ}$. The increases in high frequency noise for IPR* $=0.82$ over that of the no injection case in frequency bands greater than, or equal to, $3160 \mathrm{~Hz}$ and at aft observation angles were not observed in the data for a forward flight Mach number of 0.10 .

The sideline directivities for the 12A, 8A, and baseline nozzles for a forward flight Mach number of 0.10 and an IPR $^{*}=0.45$ are compared in Fig. 11. The baseline nozzle is the 12A nozzle with no injection flow. The sideline directivities for the 12A and 8A nozzles are quite similar. The acoustic spectra in Figs. $12 \mathrm{a}$ ) and b) show that the acoustic radiation at aft angles is unaffected by the number of injectors while the high frequency noise at $90^{\circ}$ observation angles increases with increasing numbers of injectors.

The sideline directivities for the 12A, 8A, and baseline nozzles for a forward flight Mach number of 0.28 and $\mathrm{IPR}^{*}=0.45$ are shown in Fig. 13. In contrast to the data for the forward flight Mach number of 0.10, no reduction in overall sound pressure level over that of the baseline nozzle is obtained for the 8A nozzle despite the enhanced mixing predicted by the numerical simulations. Differences in the acoustic radiation of the 12A and 8A nozzles are noted for observation angles of $90^{\circ}$ and $140^{\circ}$ as shown in the acoustic spectra of Figs. 14 a) and b). At a $90^{\circ}$ observation angle, increased high frequency noise over that of the baseline is observed for both fluidic chevron nozzles. The 12A nozzle produces higher sound pressure levels in frequency bands greater than, or equal to, $400 \mathrm{~Hz}$ than the 8A nozzle. At aft observation angles, the 12A nozzle has a greater impact on low frequency noise than the 8A nozzle. The number of injectors appears to affect the high frequency noise radiated at $90^{\circ}$ observation angles and the low frequency noise radiated at aft observation angles.

The acoustic spectra for the 12A and 8A nozzles with equivalent injection mass flow rates are shown in Figs. 15 a) and b). The acoustic radiation at aft angles for both nozzles is quite similar when compared on an equal injection mass flow rate basis. The high frequency components of the spectra at an observation angle of $90^{\circ}$ for the $12 \mathrm{~A}$ and 8A nozzles in Fig. 15 a) are similar although the 12A nozzle produces sound pressure levels that are higher than that of the $8 \mathrm{~A}$ nozzle for frequency bands greater than, or equal to, $800 \mathrm{~Hz}$. Evidently the high frequency radiation at $90^{\circ}$ is affected by the injection mass flow, IPR*, and number of injectors. Since the estimated injected momentum of the $12 \mathrm{~A}$ and $8 \mathrm{~A}$ nozzles at equal injection mass flow rates is quite similar, the good agreement of the data in Fig. $15 \mathrm{~b}$ ) may be the result of equivalent momentum injection for the alternating nozzles. The injected momentum has been shown to be important in the reduction of jet noise at the peak jet noise angle when using microjet injection ${ }^{6}$.

The acoustic spectra for the 12A, 8A, and $6 \mathrm{I}$ nozzles at equal injection mass flows are compared to that of the baseline nozzle in Figs. 16 a) and b). At an observation angle of $90^{\circ}$, the inflow nozzle, 6I, produces the lowest sound pressure levels at frequencies below $500 \mathrm{~Hz}$ of all nozzles tested. All fluidic chevron nozzles resulted in increases in high frequency noise over that of the baseline nozzle. For the aft observation angle, $140^{\circ}$, the inflow 
nozzle produced greater reductions in low frequency noise than the alternating fluidic chevrons despite the improved mixing characteristics of the alternating injectors over that of the inflow injectors.

The narrowband acoustic spectra for the 12A and 8A nozzles with no core or fan flow are compared to the spectra produced at takeoff conditions without the injectors operating in Figs. 17 a) and b). Equal injection mass flow rates are used for the 12A and 8A nozzles in the figure. As shown in the figure, the noise produced by the injectors is below that of the baseline nozzle for both forward flight Mach numbers and an observation angle of $90^{\circ}$. Since fluidic chevrons increase high frequency noise over that of the baseline nozzle at these normalized injection pressure ratios, the increased noise cannot be attributed to the noise produced by the injection flow alone. The changes in plume mixing characteristics and shear layer development that result from injecting air near the trailing edges of the core nozzle result in changes in high frequency acoustic radiation. At an observation angle of $140^{\circ}$, the injector noise begins to approach that of the baseline nozzle and a forward flight number of 0.28 for frequencies near $17,000 \mathrm{~Hz}$. However, little change in high frequency noise at aft observation angles is associated with the alternating injectors.

\section{Discussion and Conclusions}

Numerical simulations of fluidic chevrons show that enhanced mixing can be achieved through fluidic injection. The rate of mixing as measured by streamwise centerline total temperature decay, is greater for alternating injectors than for inflow injectors. The number of injectors, injection mass flow rate, and injection pressure ratio affect the mixing rate. The alternating fluidic chevron producing the least mixing (12A nozzle) results in the greatest acoustic suppression at aft observation angles and a forward flight Mach number of 0.28 of the two alternating nozzles tested. However, the 12A nozzle also results in elevated high frequency noise at a $90^{\circ}$ observation angle for both forward flight Mach numbers used in the experiments. Although the mixing associated with the inflow fluidic chevron (the 6 I nozzle) was significantly less than either that of the 12A or 8A nozzle, the $6 \mathrm{I}$ nozzle resulted in better acoustic characteristics than any of the alternating fluidic chevrons tested. Evidently, mixing rate alone cannot be used to determine the acoustic characteristics of the fluidic chevrons. The number of injectors, injection pressure ratio, and injection mass flow rate appear to be key parameters in controlling the acoustic radiation of the fluidic chevrons.

The acoustic performance of the alternating chevrons is impacted by the forward flight Mach number. Higher injection pressure ratios are required for overall noise reduction at the peak jet noise angle for a forward flight Mach number of 0.28 than for a forward flight Mach number of 0.10. At aft observation angles and a forward flight Mach number of 0.10 , the $12 \mathrm{~A}$ and $8 \mathrm{~A}$ nozzles produced similar acoustic spectra. The forward flight Mach number has a significant impact on the high frequency noise radiated at a $90^{\circ}$ observation angle when fluidic chevrons are used. Greater increases in high frequency noise radiated at $90^{\circ}$ are associated with alternating injection at a forward flight Mach number of 0.28 than at a forward flight Mach number of 0.10. The injectors may result in increases in high frequency turbulence close to the nozzle exit that have a more significant impact on the generation of high frequency noise for a forward flight Mach number of 0.28 than a forward flight Mach number of 0.10 . Since the shear rates for the fan stream - free stream interface are lower for a forward flight Mach number of 0.28 than 0.10, the high frequency components of the turbulence may become more important in the overall sound field for the higher Mach number. Additional flow measurements are needed to properly characterize the flow produced by the fluidic chevrons. 


\section{Acknowledgments}

The work presented here was funded through Goodrich Aerostructures, NASA'a Quiet Aircraft Technology Project, and the Federal Aviation Administration. Jacky Yu and Harry Haskin are also acknowledged for leading the mechanical design of the model system. The authors wish to thank the Jet Noise Laboratory staff at NASA Langley Research Center for their many contributions to the planning, operation, and data processing required for the experiment.

\section{References}

${ }^{1}$ Janarden, B. A., Hoff, G. E., Barter, J. W., Martens, S., Gliebe, P. R., Mengle, V., and Dalton, W. N., “AST critical propulsion and noise reduction technologies for future commercial subsonic engines," NASA/CR-2000-210039, 2000.

${ }^{2}$ Saiyed, N. and Bridges, J., "Tabs and mixers for reducing low bypass ratio jet noise,” AIAA-99-1986, Bellevue, WA, 1999.

${ }^{3}$ Martens, S. “Jet noise reduction technology development at GE Aircraft Engines,” ICAS 2002 Congress, pp. 842.1 - 842.10.

${ }^{4}$ Kenzakowski, D. C., Shipman, J., Dash, S. M., Bridges, J. E., and Saiyed, N. H., "turbulence model study of laboratory jets with mixing enhancements for noise reduction,” AIAA-2000-0219, Reno, NV, 2000.

${ }^{5}$ Greska, B., Krothapalli, A., Burnside, N., and Horne, W., "High-speed jet noise reduction using microjets on a jet engine,” AIAA-2004-2969, Manchester, U.K., 2004.

${ }^{6}$ Greska, B., and Krothapalli, A., “Jet noise reduction using aqueous microjet injection,” AIAA-2004-2971, Manchester, U.K., 2004.

${ }^{7}$ Kinzie, K., Henderson, B., Whitmire, J., and Abeysinghe, A., "Fluidic chevrons for jet noise reduction," proceedings of The 2004 International Symposium on Active Control of Sound and Vibration (ACTIVE 04), INCE/USA, Williamsburg, VA, 2004.

${ }^{8}$ Shields, F. D. and Bass, H. E., “A Study of Atmospheric Absorption of High Frequency Noise and Application to Fractional Octave Bands of Noise,” NASA/CR 2760, 1976.

${ }^{10}$ Henderson, B.S., Kinzie, K.W., and Haskin, H.H., “The Effect of Nozzle Trailing Edge on Jet Noise,” AIAA-2004-2948, Manchester, U.K., 2004.

${ }^{9}$ Amiet, R.K., "Correction of Open Jet Wind Tunnel Measurements for Shear Layer Refraction”, AIAA-77-54, 1977.

${ }^{11}$ Pao, S.P, and Abdol-Hamid, K.S., "Numerical Simulatoin of Jet Aerodynamics Using the Three-Dimensional NavierStokes Code PAB3D,” NASA TP 3596, 1996. 

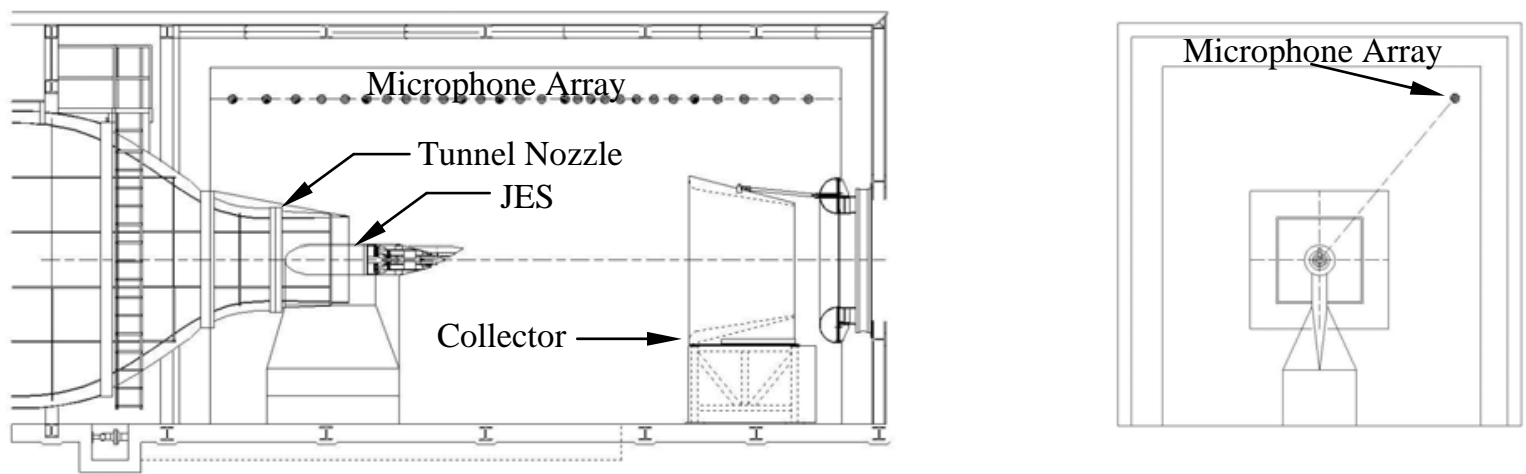

Figure 1. A schematic of the Low Speed Aeroacoustics Wind Tunnel (LSAWT) and the Jet Engine Simulator (JES).

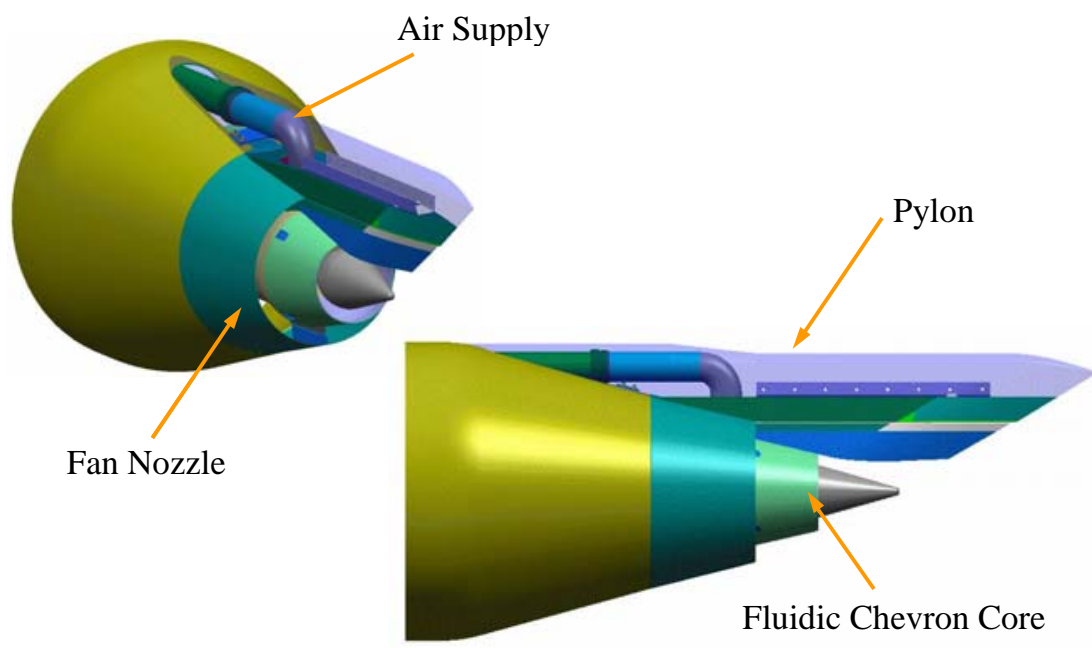

Figure 2. A schematic of the bypass ratio 5 model system used in the experiments.

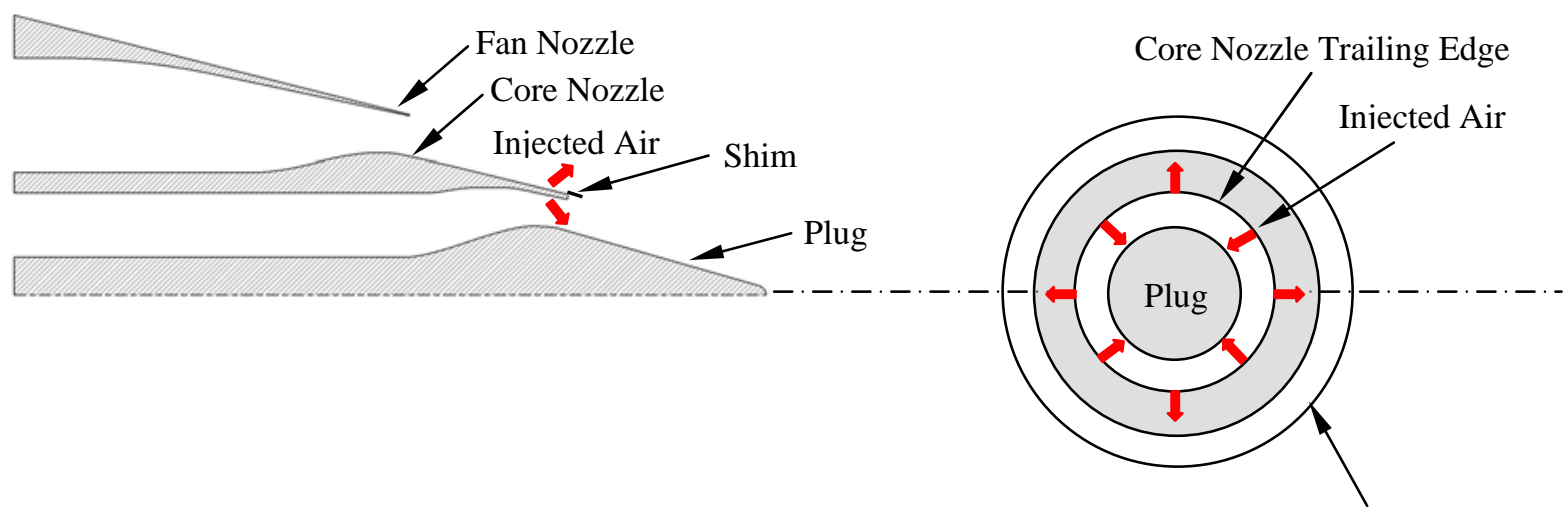

Fan Nozzle Trailing Edge

Figure 3. A schematic of the $8 \mathrm{~A}$ air injection nozzle. 

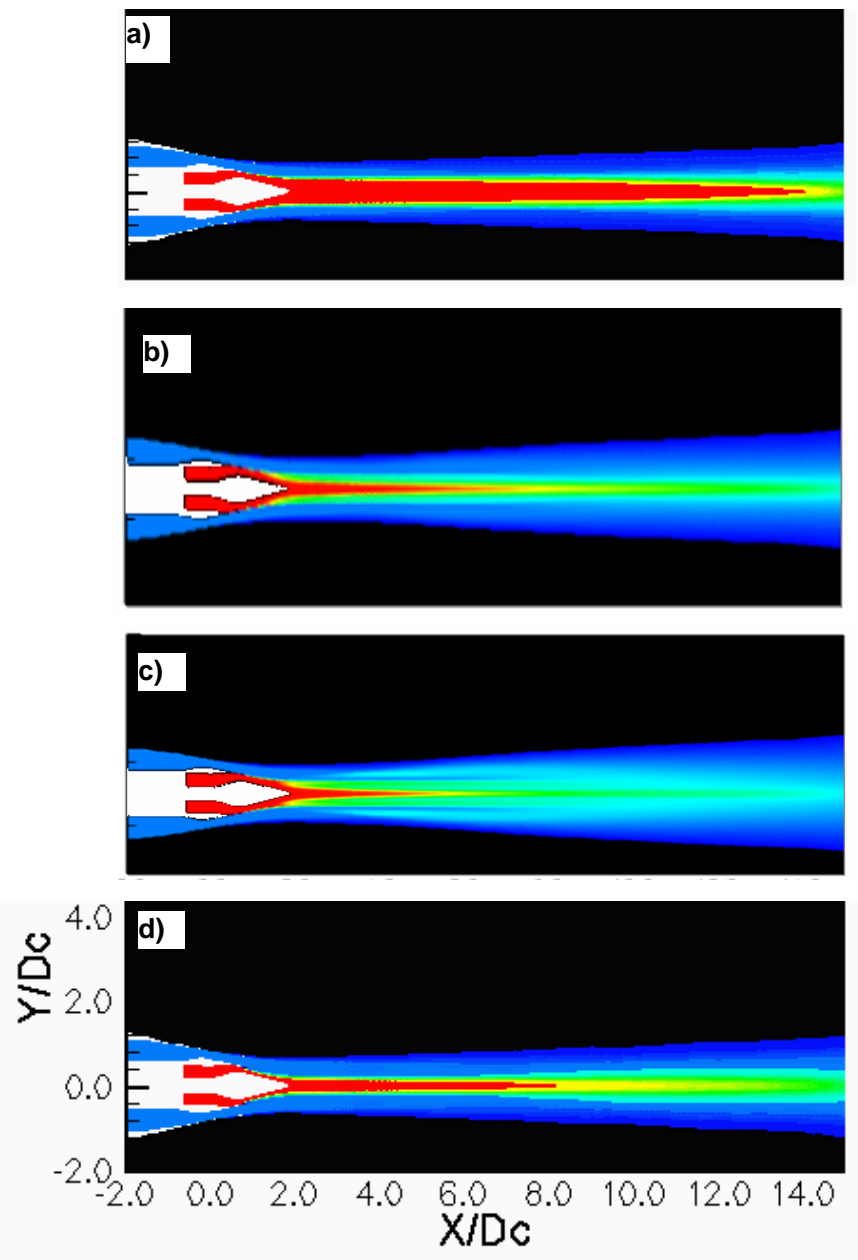

Figure 4. The centerline symmetry plane contour plots of total temperature from CFD simulations obtained at a forward flight Mach number of 0.28 and $I^{*} *=0.36$ for a) the baseline nozzle, b) the $12 \mathrm{~A}$ nozzle, $c$ ) the $8 \mathrm{~A}$ nozzle, and d) the $6 \mathrm{I}$ nozzle.

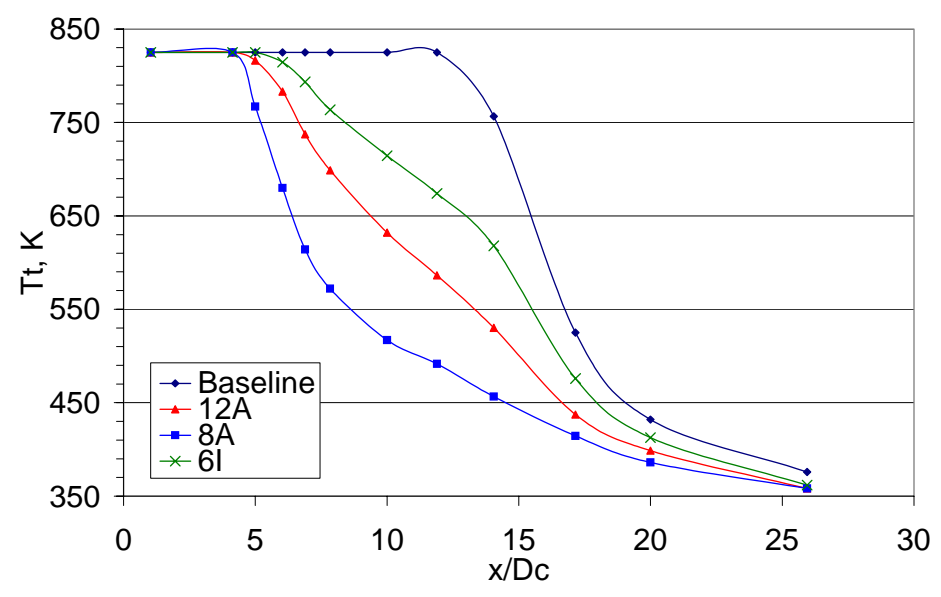

Figure 5. The predicted centerline total temperatures as a function of axial distance downstream of the fan nozzle exit obtained at a forward flight Mach number of 0.28 and IPR $*=0.36$. The axial distance, $x$, has been normalized by the core nozzle diameter, $\mathrm{Dc}$. 


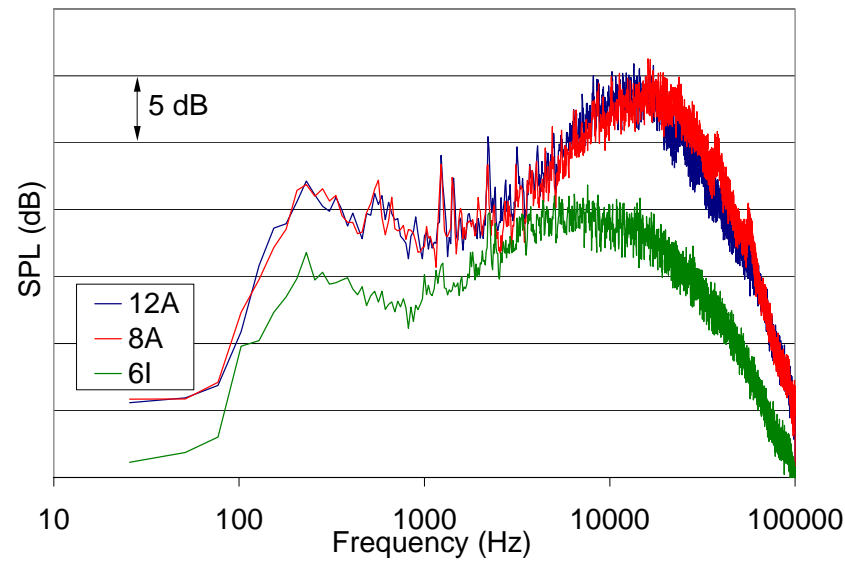

a)

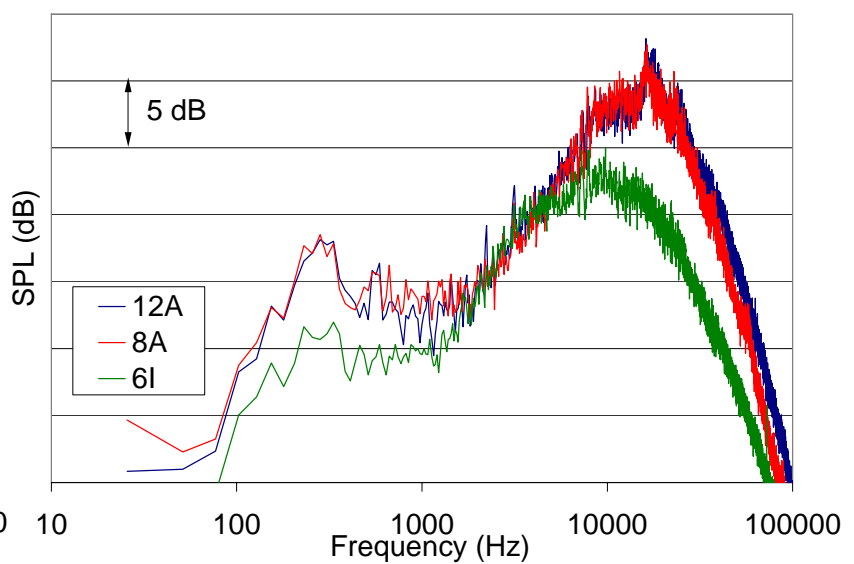

b)

Figure 6. A comparison of the injector noise for the $12 \mathrm{~A}, 8 \mathrm{~A}$, and $6 \mathrm{I}$ nozzles at an $\mathrm{IPR}^{*}=0.36$ and observation angles equal to a) $90^{\circ}$ and b) $140^{\circ}$.

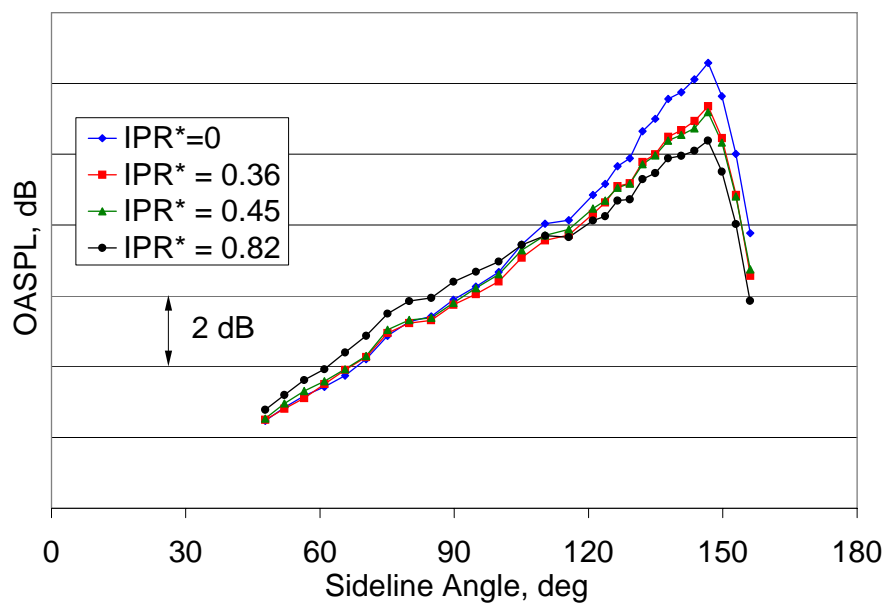

Figure 7. The sideline directivities for the 12A nozzle at a forward flight Mach number of 0.10.

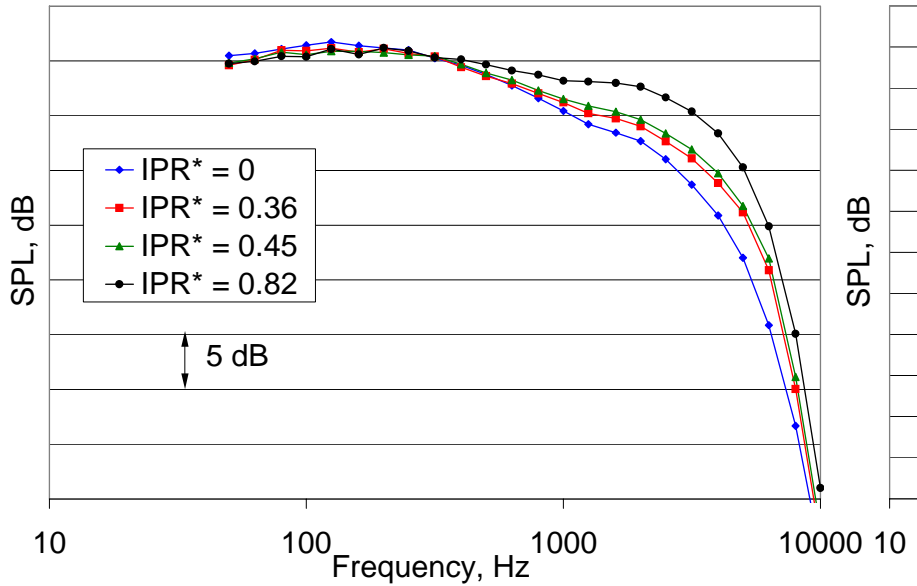

a)

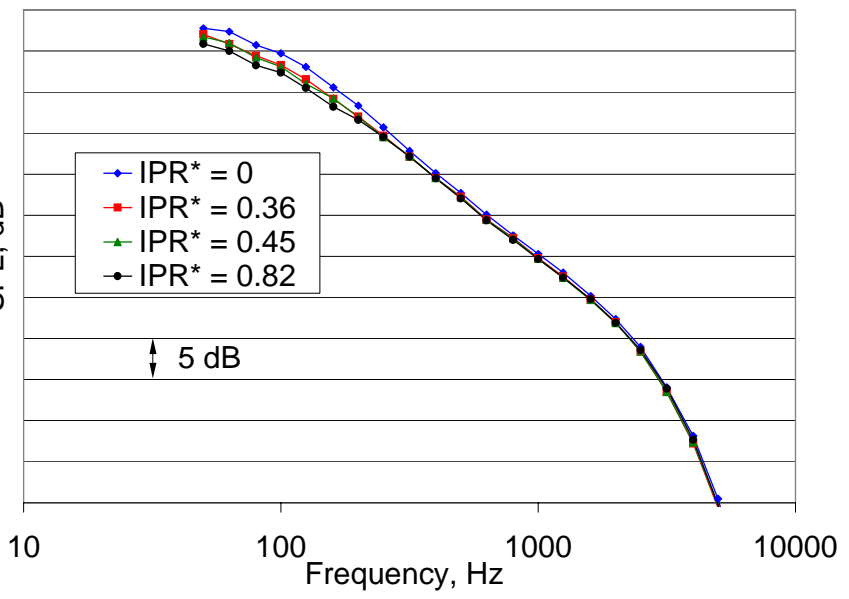

b)

Figure 8. The acoustic spectra for the 12A nozzle at a forward flight Mach number of 0.10 and observation angles equal to a) $90^{\circ}$ and b) $145^{\circ}$. 


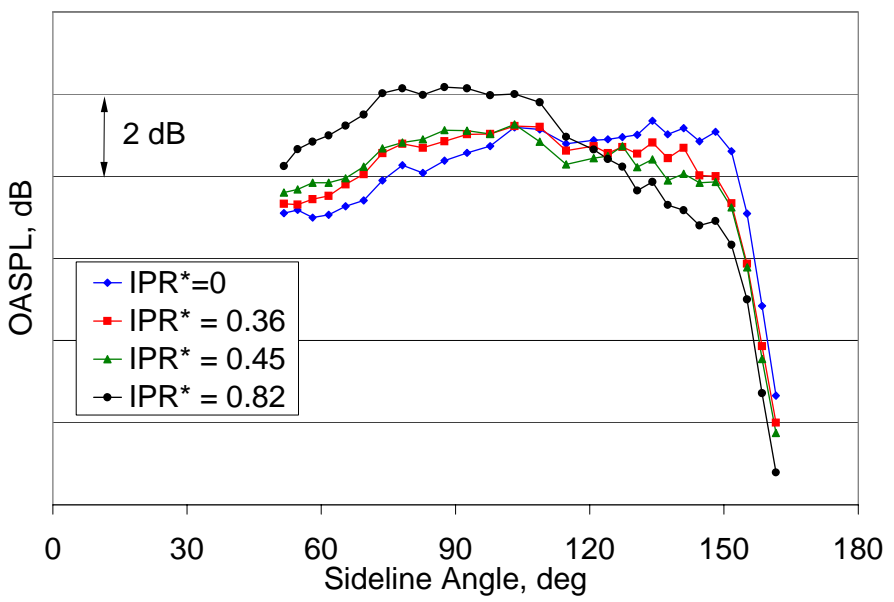

Figure 9. The sideline directivities for the 12A nozzle at a forward flight Mach number of 0.28 .
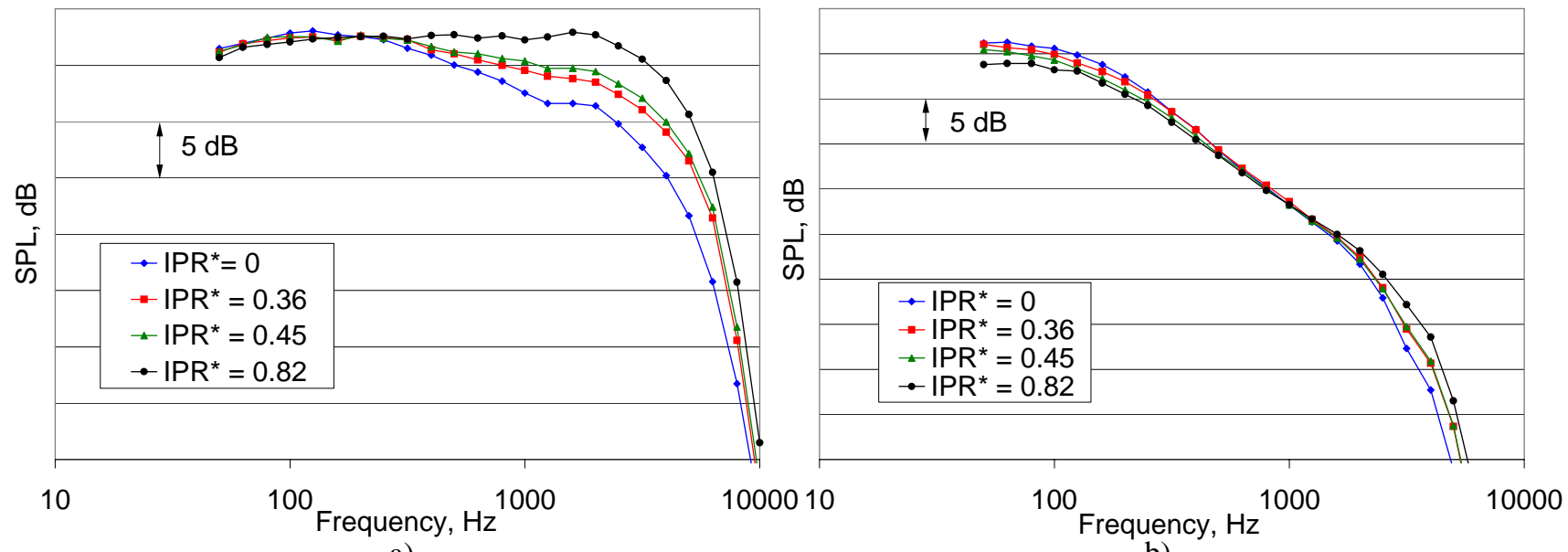

a)

b)

Figure 10. The acoustic spectra for the 12A nozzle at a forward flight Mach number of 0.28 and observation angles equal to a) $90^{\circ}$ and b) $140^{\circ}$.

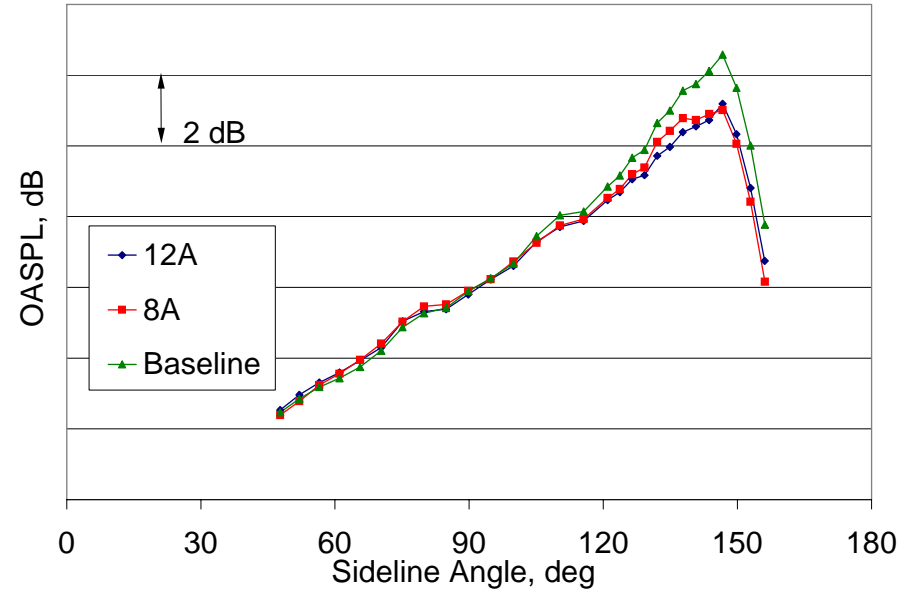

Figure 11. The sideline directivities for the 12A and 8A nozzles at a forward flight Mach number of 0.10 and IPR* $=0.45$. The baseline nozzle is the 12A nozzle with no injection flow. 


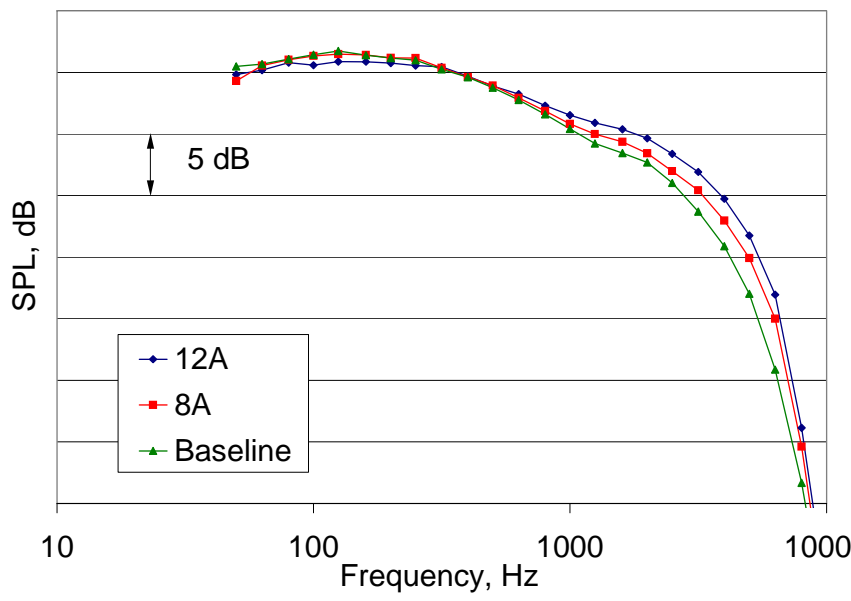

a)

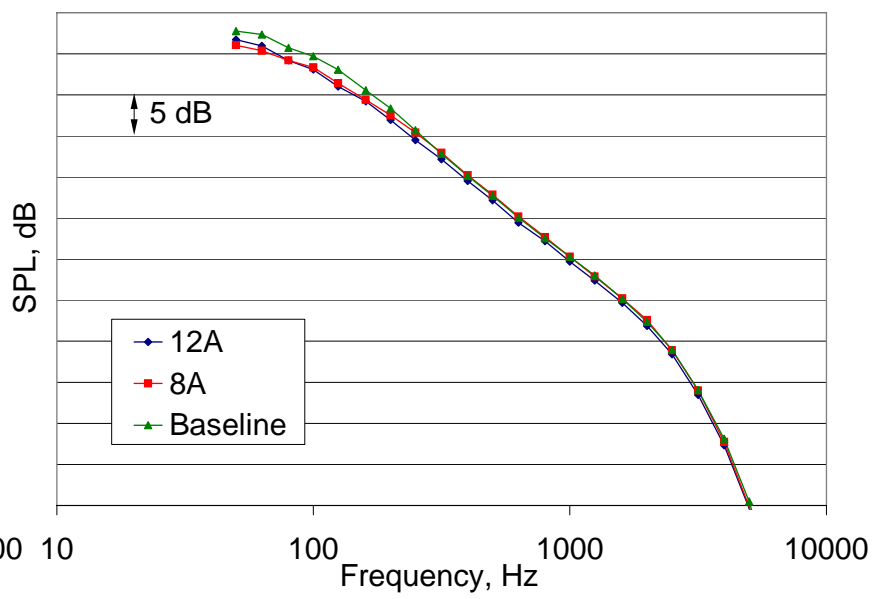

b)

Figure 12. The acoustic spectra for the $12 \mathrm{~A}$ and $8 \mathrm{~A}$ nozzles at a forward flight Mach number of $0.10 \mathrm{IPR} *=$ 0.45. The observation angles are a) $90^{\circ}$ and b) $145^{\circ}$.

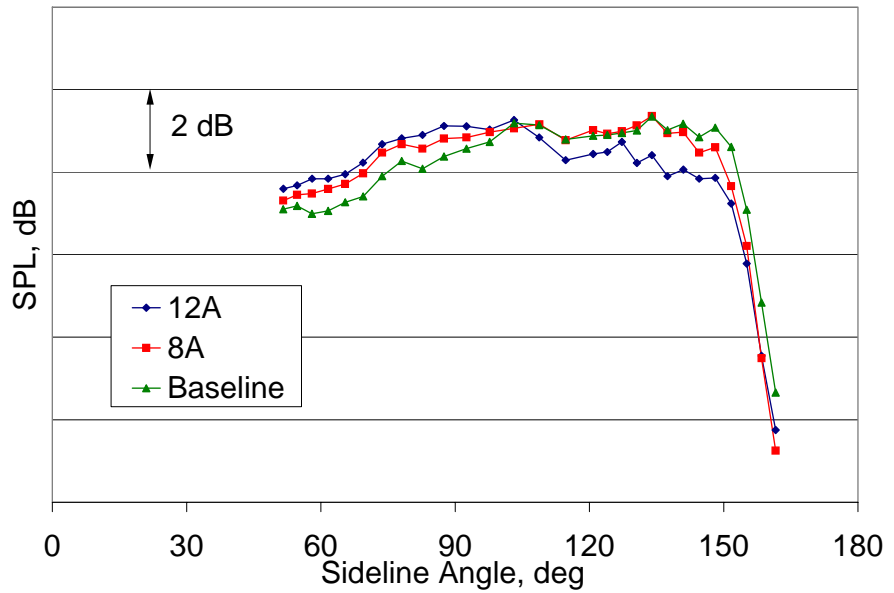

Figure 13. The sideline directivities for the $12 \mathrm{~A}$ and $8 \mathrm{~A}$ nozzles at a forward flight Mach number of 0.28 and an IPR* $=0.45$.

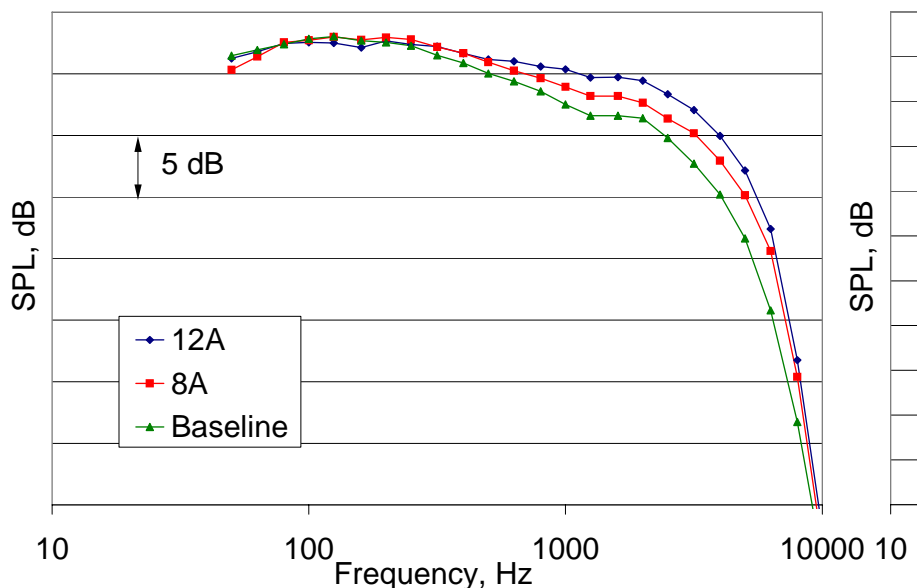

a)

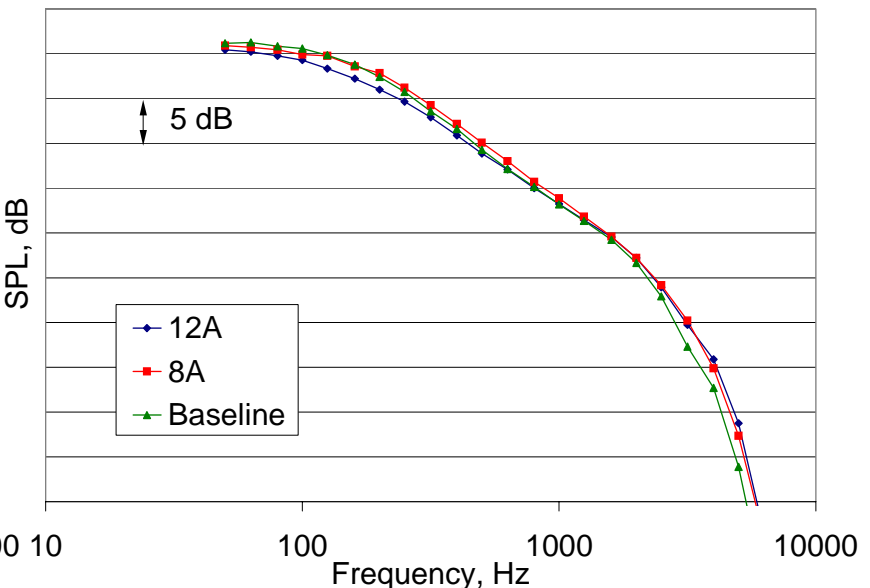

b)

Figure 14. The acoustic spectra for the $12 \mathrm{~A}$ and $8 \mathrm{~A}$ nozzles at a forward flight Mach number of 0.28 and IPR* $=0.45$. The observations angles are a) $90^{\circ}$ and b) $140^{\circ}$. 


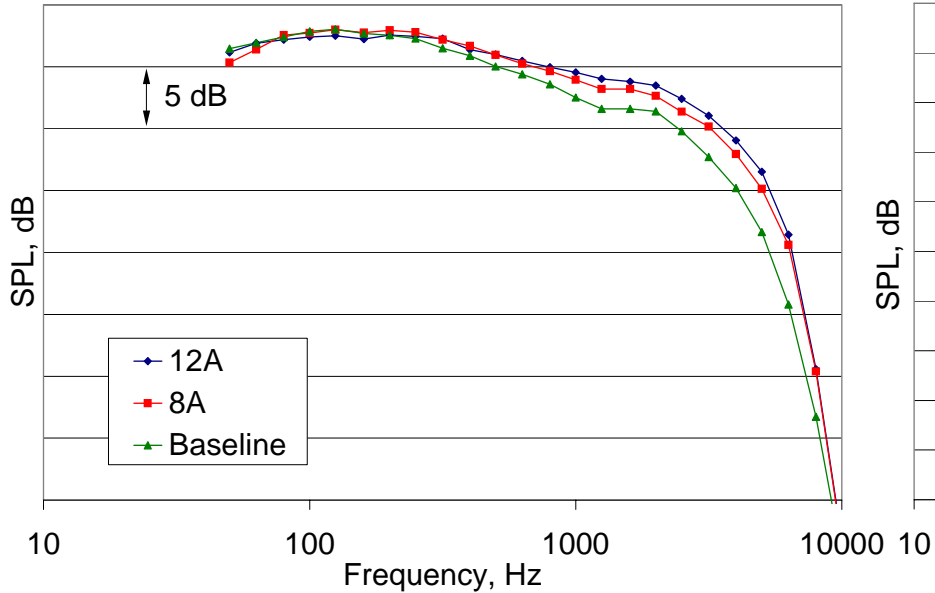

a)

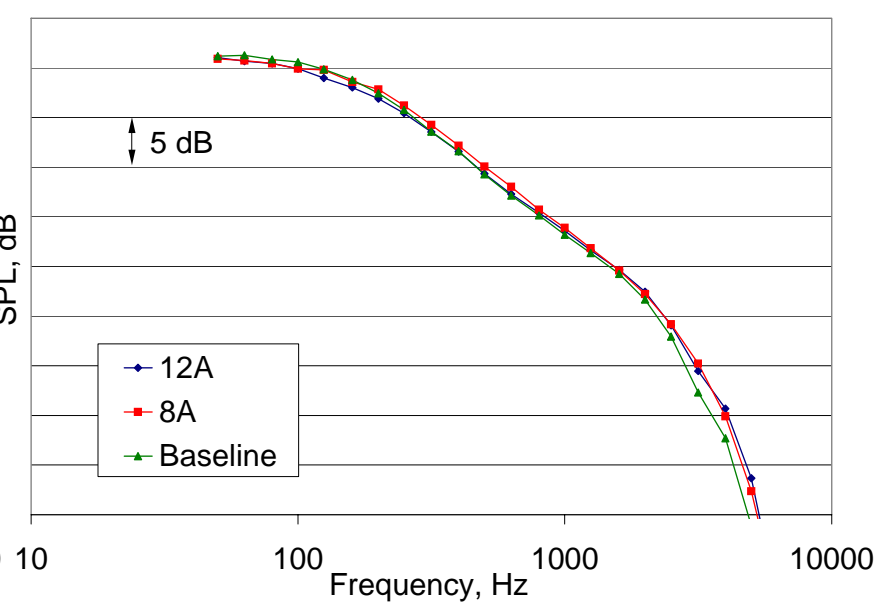

b)

Figure 15. The acoustic spectra for the $12 \mathrm{~A}$ and $8 \mathrm{~A}$ nozzles at a forward flight Mach number of 0.28 and observation angles of a) $90^{\circ}$ and b) $140^{\circ}$. Equal injection mass flow rates are used for the 12A and 8A nozzles. The normalized injection pressure ratios for the $12 \mathrm{~A}$ and $8 \mathrm{~A}$ nozzles are 0.36 and 0.45 , respectively.

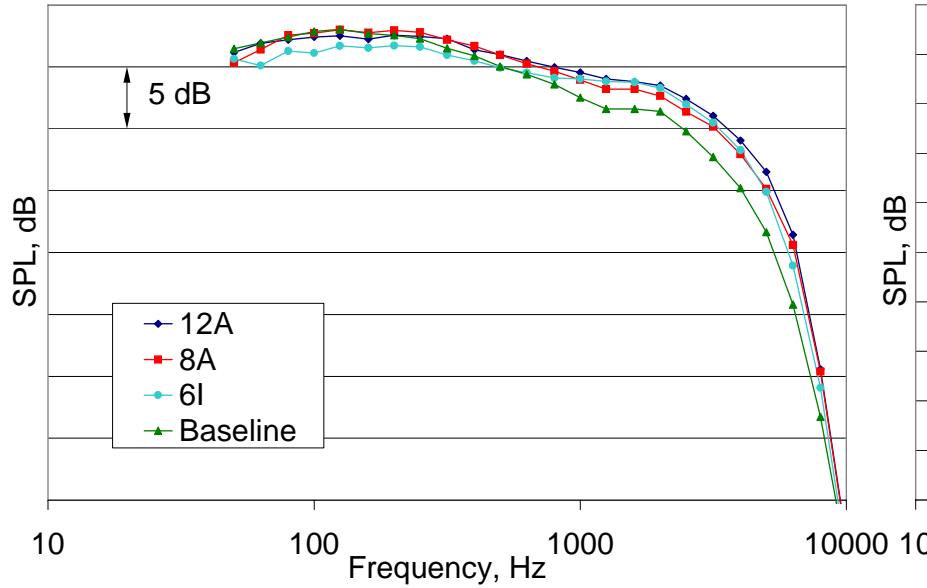

a)

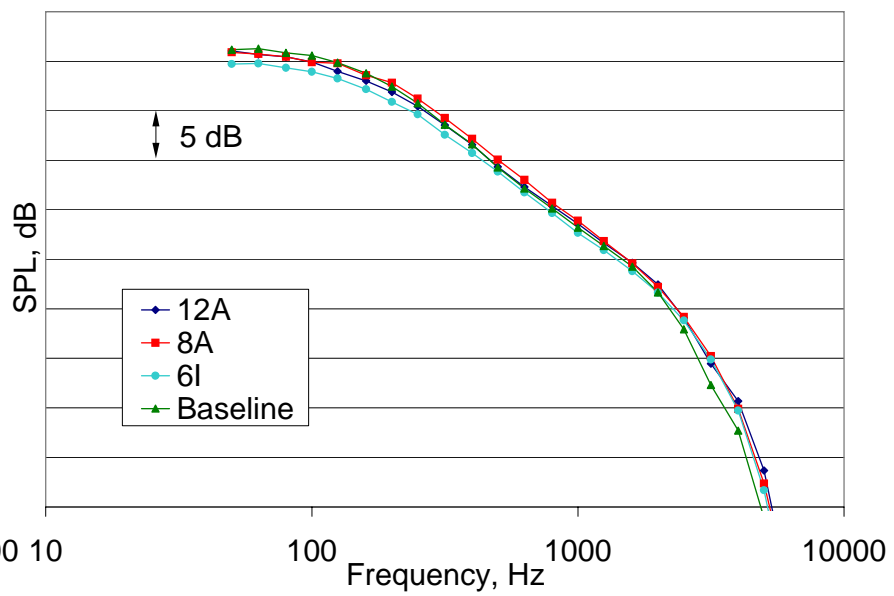

b)

Figure 16. The acoustic spectra for the 12A, 8A, and $8 \mathrm{I}$ nozzles at a forward flight Mach number of 0.28 and observation angles of a) $90^{\circ}$ and b) $140^{\circ}$. Equal injection mass flow rates are used for the $12 \mathrm{~A}, \mathbf{8 A}$, and $6 \mathrm{I}$ nozzles. The normalized injection pressure ratios for the $12 \mathrm{~A}, 8 \mathrm{~A}$, and $6 \mathrm{I}$ nozzles are $0.36,0.45$, and 0.64 , respectively. 


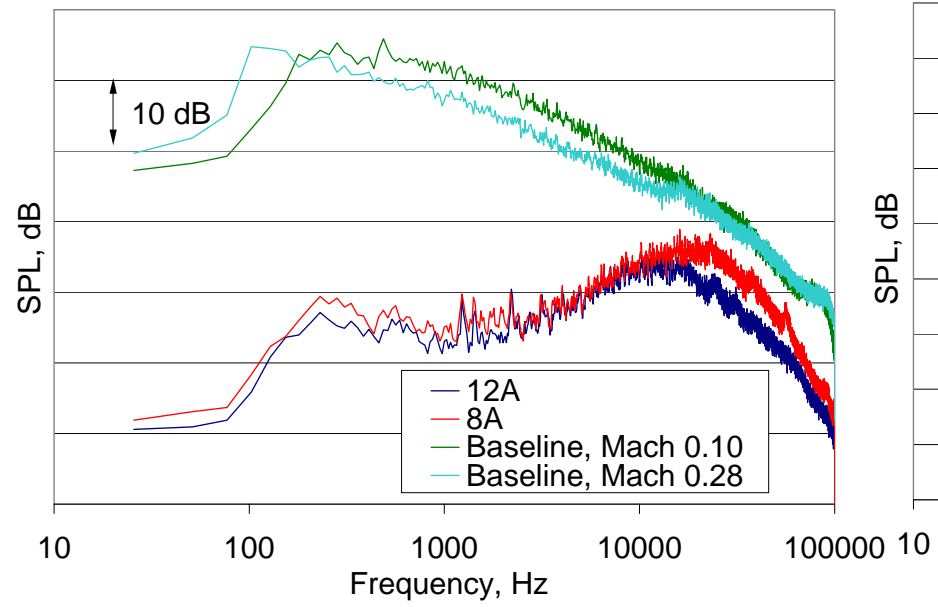

a)

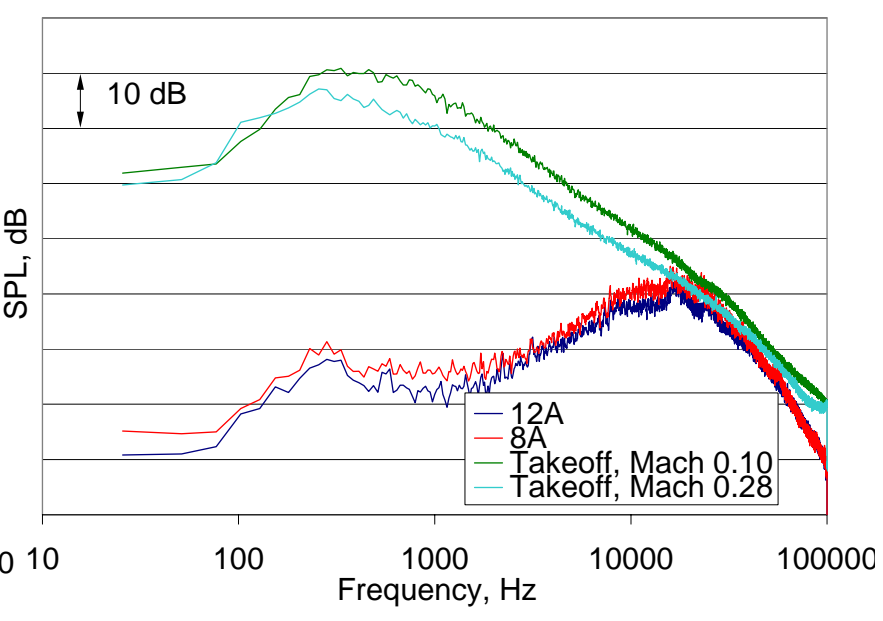

b)

Figure 17. The acoustic spectra at observation angles of a) $90^{\circ}$ and b) $140^{\circ}$. The data are for the injectors alone (12A and $8 \mathrm{~A}$ nozzles) with equal mass injection flow rates and for the baseline conditions with no injection. The normalized injection pressure ratios for the $12 \mathrm{~A}$ and $8 \mathrm{~A}$ nozzle are 0.36 and 0.45 , respectively. 